\title{
Weakly spectrally complete pair of matrices
}

\section{Laura Iglésias \& Fernando C. Silva}

To cite this article: Laura Iglésias \& Fernando C. Silva (2016) Weakly spectrally complete pair of matrices, Linear and Multilinear Algebra, 64:5, 942-950, DOI: 10.1080/03081087.2015.1067668

To link to this article: http://dx.doi.org/10.1080/03081087.2015.1067668

\section{曲 Published online: 24 Jul 2015.}

Submit your article to this journal $\pi$

Џll Article views: 21

Q View related articles ¿

View Crossmark data ¿ 


\title{
Weakly spectrally complete pair of matrices
}

\author{
Laura Iglésias $^{\mathrm{ab} *}$ and Fernando C. Silva ${ }^{\mathrm{bc}}$ \\ ${ }^{a}$ Área Departamental de Matemática, Instituto Superior de Engenharia de Lisboa-ISEL, Lisboa, \\ Portugal $;{ }^{b}$ Centro de Análise Funcional, Estruturas Lineares e Aplicações (CEAFEL), \\ Universidade de Lisboa, Lisboa, Portugal; ${ }^{c}$ Departamento de Matemática, Faculdade de Ciências \\ da Universidade de Lisboa, Lisboa, Portugal \\ Communicated by J.F. Queiró
}

(Received 20 April 2015; accepted 17 June 2015)

\begin{abstract}
Let $A$ and $B$ be $n \times n$ matrices over an algebraically closed field $F$. Let $c_{1}, \ldots, c_{n}$ be elements of $F$ such that $\operatorname{det}(A B)=c_{1} \ldots c_{n}$ and $\#\left\{i \in\{1, \ldots, n\}: c_{i} \neq 0\right\} \leq$ $\min \{\operatorname{rank}(A), \operatorname{rank}(B)\}$. We give necessary and sufficient condition for the existence of matrices $A^{\prime}$ and $B^{\prime}$ similar to $A$ and $B$, respectively, such that $A^{\prime} B^{\prime}$ has eigenvalues $c_{1}, \ldots, c_{n}$.
\end{abstract}

Keywords: eigenvalues; invariant polynomials; factorization of matrices

AMS Subject Classifications: 15A18; 15A23

Let $F$ be an algebraically closed field and $A, B \in F^{n \times n}$, where $n \geq 2$.

In this paper, we study the possible eigenvalues of the product $A^{\prime} B^{\prime}$, where $A^{\prime}, B^{\prime} \in$ $F^{n \times n}$ are matrices similar to $A, B$, respectively. If $c_{1}, \ldots, c_{n} \in F$ are the eigenvalues of $A^{\prime} B^{\prime}$ then there are two conditions that the eigenvalues must satisfy:

$$
\begin{aligned}
\operatorname{det}(A B) & =c_{1} \ldots c_{n}, \\
\#\left\{i \in\{1, \ldots, n\}: c_{i} \neq 0\right\} & \leq \min \{\operatorname{rank}(A), \operatorname{rank}(B)\} .
\end{aligned}
$$

The pair $(A, B)$ is spectrally complete, if for every sequence $c_{1}, \ldots, c_{n} \in F$ such that (1) is satisfied, there exist matrices $A^{\prime}, B^{\prime} \in F^{n \times n}$ similar to $A, B$, respectively, such that $A^{\prime} B^{\prime}$ has eigenvalues $c_{1}, \ldots, c_{n}$.

A complete description of the spectrally complete pair of matrices was given in [1], and previously, was given in [2] for the nonsingular case. The concept of spectral completeness was introduced in [3] in order to study the possible eigenvalues of the sum of matrices.

The pair $(A, B)$ is said to be weakly spectrally complete if, for every sequence $c_{1}, \ldots$, $c_{n} \in F$ such that (1) and (2) are satisfied, there exist matrices $A^{\prime}, B^{\prime}$ similar to $A, B$, respectively, such that $A^{\prime} B^{\prime}$ has eigenvalues $c_{1}, \ldots, c_{n}$.

Note that there exist $A^{\prime}, B^{\prime}$ similar to $A, B$, respectively, such that $A^{\prime} B^{\prime}$ has eigenvalues $c_{1}, \ldots, c_{n}$ if and only if there exists $A^{\prime \prime}$ similar to $A$ such that $A^{\prime \prime} B$ has eigenvalues $c_{1}, \ldots, c_{n}$ if and only if there exists $B^{\prime \prime}$ similar to $B$ such that $A B^{\prime \prime}$ has eigenvalues $c_{1}, \ldots, c_{n}$.

\footnotetext{
*Corresponding author. Email: lazevedo@adm.isel.pt
} 
Given a monic polynomial $f(x)=x^{n}+a_{n-1} x^{n-1}+\cdots+a_{1} x+a_{0}$, we denote by $C(f)$ the companion matrix of $f$ :

$$
C(f)=\left[\begin{array}{ccccc}
0 & 1 & & & 0 \\
& \cdot & \cdot & . & \\
0 & & \cdot & 0 & 1 \\
-a_{0} & -a_{1} & \ldots & -a_{n-2} & -a_{n-1}
\end{array}\right] \in F^{n \times n} .
$$

We denote by $i(A)$ the number of nonconstant invariant polynomials of $A$. We make the convention that the invariant polynomials are always monic. If $\alpha_{1}|\ldots| \alpha_{n}$ are the invariant polynomials of $A$, then $A$ is similar to $C\left(\alpha_{n-i(A)+1}\right) \oplus \cdots \oplus C\left(\alpha_{n}\right)$.

We say that $\lambda \in F$ is a primary eigenvalue of $A$ if $\lambda$ is a eigenvalue of $\alpha_{n-i(A)+1}$. Note that if $\lambda$ is a primary eigenvalue of $A$, then $\operatorname{rank}\left(A-\lambda I_{n}\right)=n-i(A)$.

If $C=\left[c_{i, j}\right] \in F^{n \times n}$ is a matrix such that $c_{i, j}=0$ if $j>i+1$, we denote by $\chi(C)$ the number of indices $i \in\{1, \ldots, n-1\}$ such that $c_{i, i+1} \neq 0$. We have $i(C) \leq n-\chi(C)$.

The next theorem is our main theorem:

Theorem 1 Let $\alpha_{1}|\ldots| \alpha_{n}$ and $\beta_{1}|\ldots| \beta_{n}$ be the invariant polynomials of $A$ and $B$, respectively. The pair $(A, B)$ is weakly spectrally complete if and only if the following are satisfied:

$$
\begin{gathered}
\text { If } i(A)+i(B)>n \text { and } \alpha_{n-i(A)+1}(x)=x-\lambda, \text { with } \lambda \in F \backslash\{0\}, \text { then } \\
\beta_{1}(x) \ldots \beta_{i(A)}(x)=x^{i(A)+i(B)-n}
\end{gathered}
$$

If $i(A)+i(B)>n$ and $\beta_{n-i(B)+1}(x)=x-\mu$, with $\mu \in F \backslash\{0\}$, then

$$
\alpha_{1}(x) \ldots \alpha_{i(B)}(x)=x^{i(A)+i(B)-n} ;
$$

(1.3) At least one of the following conditions holds:

- $n=2$,

- $\operatorname{deg}\left(\alpha_{n}\right) \neq 2$,

- $\operatorname{deg}\left(\beta_{n}\right) \neq 2$,

- $i(A) \leq i(B)$ and 0 is a primary eigenvalue of $B$,

- $\quad i(B) \leq i(A)$ and 0 is a primary eigenvalue of $A$.

Lemмa 2 If the pair $(A, B)$ is weakly spectrally complete, then (1.1) is satisfied.

Proof Suppose that $(A, B)$ is weakly spectrally complete, $i(A)+i(B)>n$ and $\alpha_{n-i(A)+1}(x)=x-\lambda$, with $\lambda \in F \backslash\{0\}$. If $A$ and $B$ are nonsingular then for every sequence $c_{1}, \ldots, c_{n} \in F$ such that $\operatorname{det}(A B)=c_{1} \ldots c_{n}$, there exist matrices $A^{\prime}, B^{\prime} \in F^{n \times n}$ similar to $A, B$, respectively, such that $A^{\prime} B^{\prime}$ has eigenvalues $c_{1}, \ldots, c_{n}$ and then the pair $(A, B)$ is spectrally complete. By Theorem 1 of [2], we have $i(A)+i(B) \leq n$, which is impossible. Then one of the matrices $A, B$ is singular and there exists a matrix $B^{\prime} \in F^{n \times n}$ similar to $B$ such that $A B^{\prime}$ has all its eigenvalues equal to 0 . Let $\gamma_{1}(x)|\ldots| \gamma_{n}(x)$ be the invariant polynomials of $A B^{\prime}$. Then

$$
\gamma_{1}(x) \ldots \gamma_{n}(x)=x^{n} .
$$


We have $A B^{\prime}=\lambda B^{\prime}+\left(A-\lambda I_{n}\right) B^{\prime}$. If $\beta_{1}(x)|\ldots| \beta_{n}(x)$ are the invariant polynomials of $B$ then $\beta_{1}\left(\lambda^{-1} x\right)|\ldots| \beta_{n}\left(\lambda^{-1} x\right)$ are the invariant polynomials of $\lambda B^{\prime}$. As $\lambda$ is a primary eigenvalue of $A$, we have $\operatorname{rank}\left(\left(A-\lambda I_{n}\right) B^{\prime}\right) \leq n-i(A)$, and by [4, Theorem 2], we conclude that

$$
\beta_{j}\left(\lambda^{-1} x\right) \mid \gamma_{j+n-i(A)}(x), \quad j \in\{1, \ldots, i(A)\} .
$$

Using (3) and (4), the invariant polynomials $\beta_{n-i(B)+1}(x), \ldots, \beta_{i(A)}(x)$ must be powers of $x$ and $\operatorname{rank}(B)=n-i(B)<i(A) \leq \operatorname{rank}(A)$.

Let $c_{1}=\cdots=c_{n-i(B)}=1$ and $c_{i(B)}=\cdots=c_{n}=0$. There exists a matrix $B^{\prime \prime} \in F^{n \times n}$ similar to $B$ such that $A B^{\prime \prime}$ has eigenvalues $c_{1}, \ldots, c_{n}$. Let $\delta_{1}(x)|\ldots| \delta_{n}(x)$ be the invariant polynomials of $A B^{\prime \prime}$. As in the previous argument, we have

$$
\beta_{j}\left(\lambda^{-1} x\right) \mid \delta_{j+n-i(A)}(x), \quad j \in\{1, \ldots, i(A)\} .
$$

Note that

$$
\delta_{1}(x) \ldots \delta_{n}(x)=x^{i(B)}(x-1)^{n-i(B)}
$$

and $\operatorname{rank}\left(A B^{\prime \prime}\right) \leq \operatorname{rank}\left(B^{\prime \prime}\right)=n-i(B)$, so $\delta_{n-i(B)+1}(0)=\cdots=\delta_{n}(0)=0$. Then

$$
\delta_{k}(x)=x(x-1)^{l_{k}}, \quad k \in\{n-i(B)+1, \ldots, n\},
$$

for some $l_{k} \in \mathbb{N}_{0}$. Therefore,

$$
\beta_{n-i(B)+1}(x)=\cdots=\beta_{i(A)}(x)=x
$$

and

$$
\beta_{1}(x) \ldots \beta_{i(A)}(x)=x^{i(A)+i(B)-n} .
$$

Lemma 3 If the pair $(A, B)$ is weakly spectrally complete then (1.3) is satisfied.

Proof Suppose that the pair $(A, B)$ is weakly spectrally complete and $n \neq 2, \operatorname{deg}\left(\alpha_{n}\right)=$ $\operatorname{deg}\left(\beta_{n}\right)=2$. Then $A$ and $B$ are similar to matrices of the form

$$
A^{\prime}=\left[\begin{array}{cc}
\lambda I_{i(A)} & * \\
0 & \nu I_{n-i(A)}
\end{array}\right] \text { and } B^{\prime}=\left[\begin{array}{cc}
\mu I_{i(B)} & * \\
0 & \epsilon I_{n-i(B)}
\end{array}\right],
$$

respectively, where $\lambda, v$ are the roots of $\alpha_{n}$ and $\mu, \epsilon$ are the roots of $\beta_{n}$.

Suppose that $i(A) \leq i(B)$ as the complementary case is analogous. We shall say that a sequence $c_{1}, \ldots, c_{n}$ of elements of $F$ are admissible if there exist matrices $A^{\prime}, B^{\prime}$ similar to $A, B$, respectively, such that $A^{\prime} B^{\prime}$ has eigenvalues $c_{1}, \ldots, c_{n}$.

Let $c_{1}, \ldots, c_{n} \in F$ be any admissible sequence. Using the arguments presented in the proof of Theorem 1 of [2], we deduce that there exists a permutation $\pi$ of $\{1, \ldots, n\}$ such that

$$
\begin{aligned}
c_{\pi(2 i-1)} c_{\pi(2 i)} & =\lambda \nu \mu \epsilon, & & 1 \leq i \leq n-i(B) \\
c_{\pi(j)} & =\lambda \mu, & & 2(n-i(B))<j \leq n+i(A)-i(B) \\
c_{\pi(j)} & =v \mu, & & n+i(A)-i(B)<j \leq n .
\end{aligned}
$$

If $A$ and $B$ are nonsingular, we can find a sequence $c_{1}, \ldots, c_{n} \in F$ such that $\operatorname{det}(A B)=$ $c_{1} \ldots c_{n}$ but the equalities (6)-(8) are not satisfied. 
Suppose that at least one of the matrices $A, B$ is singular. As the pair $(A, B)$ is weakly spectrally complete, the sequence of $\mathrm{n}$ zeros is admissible and should satisfy the equalities (6)-(8). Then $\lambda=v=0$ or $\mu=0$. If $\lambda=v=0$, then the sequence of $\mathrm{n}$ zeros is the only admissible sequence, which contradicts the assumption that the pair $(A, B)$ is weakly spectrally complete, $A \neq 0$ and $B \neq 0$. Therefore, $\mu=0$ and 0 is a primary eigenvalue of $B$.

Using the definition of weakly spectrally complete pair, Lemma 11 of [5] can be stated as follows:

Lemma 4 If one of the matrices $A, B$ is singular and the other is nonderogatory, then the pair $(A, B)$ is weakly spectrally complete.

Lemma 5 [1, Lemma 4] If $\min \{\operatorname{rank}(A), \operatorname{rank}(B)\} \geq n-1$, one of the matrices $A, B$ is nonderogatory and the other is nonscalar, then the pair $(A, B)$ is spectrally complete.

According to the two previous Lemmas, we have:

Lemma 6 If one of the matrices $A, B$ is nonderogatory and the other is nonscalar, then the pair $(A, B)$ is weakly spectrally complete.

Lemma 7 If $i(A)+i(B) \leq n$ and, either $n=2$ or at least one of the polynomials $\alpha_{n}, \beta_{n}$ has degree different from 2 , then $(A, B)$ is weakly spectrally complete.

Proof This proof is by induction on $n$. If $\min \{\operatorname{rank}(A), \operatorname{rank}(B)\} \geq n-1$, then, according to [1, Theorem 1], the pair $(A, B)$ is spectrally complete and then is weakly spectrally complete.

Suppose that $\min \{\operatorname{rank}(A), \operatorname{rank}(B)\}<n-1$. Suppose, without loss of generality [2, Lemma 1], that $\operatorname{rank}(A) \leq \operatorname{rank}(B)$. If $B$ is nonderogatory the result follows from Lemma 4. In particular, Lemma 4 covers the case $n \leq 3$.

Suppose that $n \geq 4$ and $B$ is derogatory. Let $c_{1}, \ldots, c_{n}$ be elements of $F$ such that $\operatorname{det}(A B)=c_{1} \ldots c_{n}$ and $\#\left\{i \in\{1, \ldots, n\}: c_{i} \neq 0\right\} \leq \min \{\operatorname{rank}(A), \operatorname{rank}(B)\}$ in order to prove that there exist matrices $A^{\prime}, B^{\prime} \in F^{n \times n}$ similar to $A, B$, respectively, such that $A^{\prime} B^{\prime}$ has eigenvalues $c_{1}, \ldots, c_{n}$. Suppose, without loss of generality, that $c_{n-1}=c_{n}=0$. If there exists $i \in\left\{\{1, \ldots, n-2\}: c_{i} \neq 0\right\}$, suppose, without loss of generality, that $c_{1} \neq 0$.

Case 1. Suppose that $c_{1} \neq 0$. The matrix $A$ is similar to the direct sum of the companion matrices of its nonconstant invariant polynomials $K=C\left(\alpha_{n}\right) \oplus \cdots \oplus C\left(\alpha_{n-i(A)+1}\right)$.

- If $\operatorname{deg}\left(\alpha_{n}\right) \geq 3$, then, according to [1, Lemma 5], $K$ is similar to a matrix of the form

$$
K^{\prime}=\left[\begin{array}{ccc}
* & * & 1 \\
* & K_{0} & 0 \\
0 & 0 & 0
\end{array}\right],
$$

where $K_{0} \in F^{(n-2) \times(n-2)}$ is a direct sum of companion matrices, $\chi\left(K_{0}\right)=\chi(K)-1$ and $\operatorname{det}\left(K_{0}\right)=0$. Moreover, if $i(A) \leq n-3$ (i.e. $\chi(K) \geq 3$ ), then $K_{0}$ has been 
chosen so that at least one of the companion matrices appearing in $K_{0}$ is of size $u \times u$, with $u \geq 3$ and then the minimum polynomial of $K_{0}$ has degree greater than 2;

- If $\operatorname{deg}\left(\alpha_{n}\right)=2$, then $C\left(\alpha_{n}\right)$ is similar to a matrix of the form

$$
\left[\begin{array}{ll}
* & 1 \\
0 & 0
\end{array}\right]
$$

and $K$ is similar to a matrix of the form

$$
K^{\prime}=\left[\begin{array}{ccc}
* & 0 & 1 \\
0 & K_{0} & 0 \\
0 & 0 & 0
\end{array}\right],
$$

where $K_{0}=C\left(\alpha_{n-i(A)+1}\right) \oplus \cdots \oplus C\left(\alpha_{n-1}\right)$. Note that $\operatorname{det} K_{0}=0$ and $\chi\left(K_{0}\right)=$ $\chi(K)-1$.

Analogously, the matrix $B$ is similar to the direct sum of the companion matrices of its nonconstant invariant polynomials $L=C\left(\beta_{n}\right) \oplus \cdots \oplus C\left(\beta_{n-i(B)+1}\right)$.

- If $\operatorname{deg}\left(\beta_{n}\right) \geq 3$, then, according to a variant of [1, Lemma 5] or a variant of [2, Lemma 4], $L$ is similar to a matrix of the form

$$
L^{\prime}=\left[\begin{array}{ccc}
0 & 0 & * \\
0 & L_{0} & * \\
c_{1} & * & *
\end{array}\right],
$$

where $L_{0} \in F^{(n-2) \times(n-2)}$ is a direct sum of companion matrices, $\operatorname{det}\left(K_{0}\right)=\operatorname{det}\left(L_{2} \oplus\right.$ $\left.\cdots \oplus L_{S}\right)$, and $\chi\left(L_{0}\right)=\chi(L)-1$. Moreover, if $i(B) \leq n-3$ (i.e. $\chi(L) \geq 3$ ), then $L_{0}$ has been chosen so that at least one of the companion matrices appearing in $L_{0}$ is of size $u \times u$, with $u \geq 3$ and then the minimum polynomial of $L_{0}$ has degree greater than 2 ;

- If $\operatorname{deg}\left(\beta_{n}\right)=2$, then $C\left(\beta_{n}\right)$ is similar to a matrix of the form

$$
\left[\begin{array}{cc}
0 & * \\
c_{1} & *
\end{array}\right]
$$

and $L$ is similar to a matrix of the form

$$
L^{\prime}=\left[\begin{array}{ccc}
0 & 0 & * \\
0 & L_{0} & 0 \\
c_{1} & 0 & *
\end{array}\right],
$$

where $L_{0}=C\left(\beta_{n-i(B)-1}\right) \oplus \cdots \oplus C\left(\beta_{n-1}\right)$. Note that $\chi\left(L_{0}\right)=\chi(L)-1$.

We have $\operatorname{det}\left(K_{0} L_{0}\right)=0=c_{2} \ldots c_{n-1}, \#\left\{i \in\{2, \ldots, n-1\}: c_{i} \neq 0\right\} \leq \min \{\operatorname{rank}(A)$, $\operatorname{rank}(B)\}-1=\min \left\{\operatorname{rank}\left(K_{0}\right), \operatorname{rank}\left(L_{0}\right)\right\}$ and $i\left(K_{0}\right)+i\left(L_{0}\right) \leq\left(n-2-\chi\left(K_{0}\right)\right)+(n-$ $\left.2-\chi\left(L_{0}\right)\right)=2 n-\chi(K)-\chi(L)-2=i(A)+i(B)-2 \leq n-2$. Now, we shall prove that either $n=4$ or at least one of the minimum polynomial of the matrices $K_{0}, L_{0}$ has degree greater than 2 . 
- If $\operatorname{deg}\left(\alpha_{n}\right) \geq 3$ and $i(A) \leq n-3$, then the minimum polynomial of the matrix $K_{0}$ has degree greater than 2 ;

- If $\operatorname{deg}\left(\beta_{n}\right) \geq 3$ and $i(B) \leq n-3$, then the minimum polynomial of the matrix $L_{0}$ has degree greater than 2 ;

- If $\operatorname{deg}\left(\alpha_{n}\right)=2$ and $i(B)>n-3$, then $(n / 2)+(n-2) \leq i(A)+i(B) \leq n$ and therefore $n=4$;

- If $\operatorname{deg}\left(\beta_{n}\right)=2$ and $i(A)>n-3$, then with similar arguments to the previous case, we conclude that $n=4$.

By the induction assumption, there exist nonsingular matrices $X_{0}, Y_{0} \in F^{(n-2) \times(n-2)}$ such that $X_{0} K_{0} X_{0}^{-1} Y_{0} L_{0} Y_{0}^{-1}$ has eigenvalues $c_{2}, \ldots, c_{n-1}$. Let $X=[1] \oplus X_{0} \oplus$ [1] and $Y=[1] \oplus Y_{0} \oplus[1]$. The matrix $X^{-1} K^{\prime} X Y^{-1} L^{\prime} Y$ has eigenvalues $c_{1}, \ldots, c_{n}$.

Case 2. Suppose that $c_{1}=0$. Then $c_{1}=\cdots=c_{n}=0$. Let $p=\min \{j \in\{n-$ $\left.i(A)+1, \ldots, n-1\}: \alpha_{j}(0)=0\right\}$. Let $\alpha_{p-1}^{\prime}(x)=\alpha_{p}(x) / x$ and $\alpha_{j}^{\prime}=\alpha_{j+1}$, for every $j \in\{1, \ldots, n-1\}$ and $j \neq p-1$.

The matrix $A$ is similar to a matrix of the form

$$
A^{\prime}=\left[\begin{array}{cc}
A_{0} & * \\
0 & 0
\end{array}\right],
$$

where $A_{0}$ has invariant polynomials $\alpha_{1}^{\prime}|\ldots| \alpha_{n-1}^{\prime}$ and $\operatorname{det}\left(A_{0}\right)=0$.

Subcase 2.1 Suppose that $i(A)+i(B)<n$ or $\operatorname{deg}\left(\beta_{n-i(B)+1}\right)=1$. Let $\mu$ be a primary eigenvalue of $B$. Let $\beta_{n-i(B)+1}^{\prime}(x)=\beta_{n-i(B)+1}(x) /(x-\mu)$. The matrix $B$ is similar to a matrix of the form

$$
B^{\prime}=\left[\begin{array}{cc}
B_{0} & * \\
0 & \mu
\end{array}\right],
$$

where

$$
\begin{array}{ll}
B_{0}=C\left(\beta_{n-i(B)+1}^{\prime}\right) \oplus C\left(\beta_{n-i(B)+2}\right) \oplus \cdots \oplus C\left(\beta_{n}\right), & \text { if } \operatorname{deg}\left(\beta_{n-i(B)+1}\right) \geq 2, \\
B_{0}=C\left(\beta_{n-i(B)+2}\right) \oplus \cdots \oplus C\left(\beta_{n}\right), & \text { if } \operatorname{deg}\left(\beta_{n-i(B)+1}\right)=1 .
\end{array}
$$

We have $i\left(A_{0}\right)+i\left(B_{0}\right) \leq n-1$ and at least one of the minimum polynomials of $A_{0}, B_{0}$ has degree greater than 2 . According to the induction assumption, $\left(A_{0}, B_{0}\right)$ is spectrally complete and it is easy to conclude that $(A, B)$ is also weakly spectrally complete.

Subcase 2.2 Suppose that $i(A)+i(B)=n$ and $\operatorname{deg}\left(\beta_{n-i(B)+1}\right) \geq 2$. Let $d=$ $\operatorname{deg}\left(\beta_{n-i(B)+1}\right)$. Analogously to the subcase 2.2 .2 of the proof of Theorem 1 of [1], we conclude that

$$
\#\left\{j \in\{1, \ldots, n\}: \operatorname{deg}\left(\alpha_{j}\right)=1\right\} \geq d-1 .
$$

Then $\alpha_{n-i(A)+1}(x)=\cdots=\alpha_{n-i(A)+d-1}(x)=x-\lambda$, where $\lambda$ is a primary eigenvalue of $A$. If $\lambda=0$, then $p=n-i(A)+1$ and $i\left(A_{0}\right)=i(A)-1$. Let $B^{\prime}$ be the matrix similar to $B$ as in the previous subcase. We have $i\left(A_{0}\right)+i\left(B_{0}\right)=n-1$ and $\alpha_{n}, \beta_{n}$ are the minimum polynomials of $A^{\prime}, B^{\prime}$. According to the induction assumption, there exist $X_{0}, Y_{0} \in F^{(n-1) \times(n-1)}$ such that $X_{0} A_{0} X_{0}^{-1} Y_{0} B_{0} Y_{0}^{-1}$ has eigenvalues $c_{1}, \ldots, c_{n-1}$. The matrix $\left(X_{0} \oplus[1]\right) A^{\prime}\left(X_{0} \oplus[1]\right)^{-1}\left(Y_{0} \oplus[1]\right) B^{\prime}\left(Y_{0} \oplus[1]\right)^{-1}$ has eigenvalues $c_{1}, \ldots, c_{n}$.

Suppose that $\lambda \neq 0$. Let $\alpha_{p-d}^{\prime \prime}(x)=\alpha_{p}(x) / x$ and $\alpha_{j}^{\prime \prime}=\alpha_{p+d}^{\prime}$, for every $j \in\{1, \ldots$, $n-d\}$ and $j \neq p-d$. The matrix $A^{\prime}$ is permutation similar to a matrix of the form

$$
\left[\begin{array}{cc}
D & * \\
0 & K_{0}
\end{array}\right]
$$


where $D=I_{d-1} \oplus[0]$ and $K_{0} \in F^{(n-d) \times(n-d)}$ has invariant polynomials $\alpha_{1}^{\prime \prime}|\ldots| \alpha_{n-d}^{\prime \prime}$. The matrix $B$ is similar to

$$
C\left(\beta_{n-i(B)+1}\right) \oplus L_{0}, \quad \text { where } \quad L_{0}=C\left(\beta_{n-i(B)+2}\right) \oplus \cdots \oplus C\left(\beta_{n}\right) .
$$

We have $i\left(K_{0}\right)+i\left(L_{0}\right)=(i(A)-d+1)+(i(B)-1)=n-d$ and $\alpha_{n}, \beta_{n}$ are the minimum polynomials of $A^{\prime}, B^{\prime}$. Then, we conclude that $\left(K_{0}, L_{0}\right)$ is weakly spectrally complete. By Lemma 4 the pair $\left(D, C\left(\beta_{n-i(B)+1}\right)\right)$ is also weakly spectrally complete. It is easy to complete the proof.

Lemma 8 If (1.1) and (1.2) are satisfied and at least one of the polynomials $\alpha_{n}, \beta_{n}$ has degree different from 2 , then the pair $(A, B)$ is weakly spectrally complete.

Proof By induction on $n$. The proof has already been done when $i(A)+i(B) \leq n$. Suppose that $i(A)+i(B)>n$. Suppose, without loss of generality [2, Lemma 1], that $i(A) \geq i(B)$. Then $\operatorname{deg}\left(\alpha_{n-i(A)+1}\right)=1$. Let $p=\#\left\{j \in\{1, \ldots, n\}: \operatorname{deg}\left(\alpha_{j}\right)=1\right\}$ and $d=\operatorname{deg}\left(\beta_{n-i(B)+1}\right)$. In order to obtain a contradiction, assume that $p<d$. Then

$$
i(A) \leq p+\frac{n-p}{2}, \quad i(B) \leq \frac{n}{d} \leq \frac{n}{p+1} .
$$

From

$$
n+1 \leq i(A)+i(B) \leq p+\frac{n-p}{2}+\frac{n}{p+1},
$$

it follows that $0 \leq h(p)$, where $h(p)=p^{2}-(n+1) p+n-2$, which is impossible because $h(1)$ and $h(n)$ are negative numbers. Therefore $p \geq d$. Let $\lambda$ be the primary eigenvalue of $A$. The matrices $A, B$ are, respectively, similar to the matrices

$$
\begin{aligned}
& A^{\prime}=\lambda I_{d} \oplus K_{0}, \quad \text { where } \quad K_{0}=C\left(\alpha_{n-i(A)+d+1}\right) \oplus \cdots \oplus C\left(\alpha_{n}\right), \\
& B^{\prime}=C\left(\beta_{n-i(B)+1}\right) \oplus L_{0} \text {, where } \quad L_{0}=C\left(\beta_{n-i(B)+2}\right) \oplus \cdots \oplus C\left(\beta_{n}\right) .
\end{aligned}
$$

Let $\alpha_{1}^{\prime}|\ldots| \alpha_{n-d}^{\prime}$ and $\beta_{1}^{\prime}|\ldots| \beta_{n-d}^{\prime}$ be the invariant polynomials of the matrices $K_{0}$ and $L_{0}$, respectively. Note that $i\left(K_{0}\right)=i(A)-d$ and $i\left(L_{0}\right)=i(B)-1$.

Case 1. Suppose that $\lambda=0$. Then $\operatorname{rank}(A)=n-i(A)$. If $p=n$, then $A=0$ and the result is trivial.

Suppose that $p<n$. If $d=1$ and $C\left(\beta_{n-i(B)+1}\right)$ is singular, then $\operatorname{rank}\left(L_{0}\right)=\operatorname{rank}(B)=$ $n-i(B) \geq n-i(A)=\operatorname{rank}(A)=\operatorname{rank}\left(K_{0}\right)$. If $d>1$ or $C\left(\beta_{n-i(B)+1}\right)$ is nonsingular, then $\operatorname{rank}\left(L_{0}\right) \geq i\left(L_{0}\right)=i(B)-1 \geq n-i(A)=\operatorname{rank}(A)=\operatorname{rank}\left(K_{0}\right)$ and $\operatorname{rank}(B) \geq$ $i(B)>n-i(A)=\operatorname{rank}(A)$.

Let $c_{1}, \ldots, c_{n} \in F$ be such that $\#\left\{i \in\{1, \ldots, n\}: c_{i} \neq 0\right\} \leq \min \{\operatorname{rank}(A), \operatorname{rank}(B)\}$ $=\operatorname{rank}(A)=n-i(A)$. Suppose without loss of generality, that $c_{1}=\cdots=c_{i(A)}=0$.

If $\beta_{(n-d)-i\left(L_{0}\right)+1}^{\prime}(x)=x-\mu$, with $\mu \in F \backslash\{0\}$, then, as $\beta_{(n-d)-i\left(L_{0}\right)+1}^{\prime}(x)=$ $\beta_{n-i(B)+2}(x)$, we have $\beta_{n-i(B)+1}(x)=\beta_{n-i(B)+2}(x)=x-\mu$. By (1.2), we have

$$
\alpha_{1}(x) \ldots \alpha_{i(B)}(x)=x^{i(A)+i(B)-n}
$$

and then

$$
\alpha_{1}^{\prime}(x) \ldots \alpha_{i\left(L_{0}\right)}^{\prime}(x)=\frac{\alpha_{1}(x) \ldots \alpha_{i(B)}(x)}{x}=x^{i(A)+i(B)-n-1}=x^{i\left(K_{0}\right)+i\left(L_{0}\right)-(n-1)} .
$$


Note that $\operatorname{rank}\left(L_{0}\right) \geq \operatorname{rank}\left(K_{0}\right)=n-i(A)$ and at least one of the polynomials $\alpha_{n-d}^{\prime}=\alpha_{n}$ and $\beta_{n-d}^{\prime}=\beta_{n}$ has degree different from 2. According to the induction assumption, there exist $X_{0}, Y_{0} \in F^{(n-d) \times(n-d)}$ such that $X_{0}^{-1} K_{0} X_{0} Y_{0}^{-1} L_{0} Y_{0}$ has eigenvalues $c_{d+1}, \ldots, c_{n}$. Consider the matrices $X=I_{d} \oplus X_{0}$ and $Y=I_{d} \oplus Y_{0}$. The matrix $X^{-1} A^{\prime} X Y^{-1} B^{\prime} Y$ has eigenvalues $c_{1}, \ldots, c_{n}$.

Case 2. Suppose that $\lambda \neq 0$. By (1.1), we have

$$
\beta_{1}(x) \ldots \beta_{i(A)}(x)=x^{i(A)+i(B)-1}
$$

which implies that

$$
\beta_{n-i(B)+1}(x)=\cdots=\beta_{i(A)}(x)=x .
$$

Note that $d=1$ and $\operatorname{rank}(B)=n-i(B)<i(A) \leq \operatorname{rank}(A)$. Let $c_{1}, \ldots, c_{n} \in F$ be such that $\#\left\{i \in\{1, \ldots, n\}: c_{i} \neq 0\right\} \leq \min \{\operatorname{rank}(A), \operatorname{rank}(B)\}=\operatorname{rank}(B)=n-i(B)$. Suppose without loss of generality, that $c_{1}=\cdots=c_{i(B)}=0$.

If $\operatorname{deg}\left(\alpha_{n-i(A)+2}\right)=1$, then

$$
\beta_{1}^{\prime}(x) \ldots \beta_{i\left(K_{0}\right)}^{\prime}(x)=\frac{\beta_{1}(x) \ldots \beta_{i(A)}(x)}{x}=x^{i(A)+i(B)-n-1}=x^{i\left(K_{0}\right)+i\left(L_{0}\right)-(n-1)} .
$$

Note that $\operatorname{rank}\left(L_{0}\right)=\operatorname{rank}(B)<\operatorname{rank}(A)=\operatorname{rank}\left(K_{0}\right)+1$ and least one of the polynomials $\alpha_{n-1}^{\prime}=\alpha_{n}$ and $\beta_{n-1}^{\prime}=\beta_{n}$ has degree different from 2. According to the induction assumption, there exist $X_{0}, Y_{0} \in F^{(n-1) \times(n-1)}$ such that $X_{0}^{-1} K_{0} X_{0} Y_{0}^{-1} L_{0} Y_{0}$ has eigenvalues $c_{2}, \ldots, c_{n}$. Consider the matrices $X=[1] \oplus X_{0}$ and $Y=[1] \oplus Y_{0}$. The matrix $X^{-1} A^{\prime} X Y^{-1} B^{\prime} Y$ has eigenvalues $c_{1}, \ldots, c_{n}$.

Lemma 9 If $n=2=\operatorname{deg}\left(\alpha_{2}\right)=\operatorname{deg}\left(\beta_{2}\right)$, then the pair $(A, B)$ is weakly spectrally complete.

Proof Follows from Lemma 6.

Lemma 10 If $\operatorname{deg}\left(\alpha_{n}\right)=\operatorname{deg}\left(\beta_{n}\right)=2, i(A) \leq i(B)$ and 0 is a primary eigenvalue of $B$, then the pair $(A, B)$ is weakly spectrally complete.

Proof Let $\lambda, v$ be the roots of $\alpha_{n}$ and $\lambda$ a primary eigenvalue of $A$. Let $0, \epsilon$ be the roots of $\beta_{n}$. The matrix $A$ is similar to

$$
A^{\prime}=\lambda I_{2 i(A)-n} \oplus \bigoplus_{i=1}^{n-i(A)} C, \text { where } C=\left[\begin{array}{ll}
\lambda & 1 \\
0 & v
\end{array}\right],
$$

and $B$ is similar to

$$
B^{\prime}=0_{2 i(B)-n} \oplus \bigoplus_{i=1}^{n-i(B)} D, \quad \text { where } \quad D=\left[\begin{array}{ll}
0 & 1 \\
0 & \epsilon
\end{array}\right] .
$$

Note that $\operatorname{rank}(B)=n-i(B) \leq n-i(A) \leq \operatorname{rank}(A)$. Let $c_{1}, \ldots, c_{n} \in F$ be such that $\#\left\{i \in\{1, \ldots, n\}: c_{i} \neq 0\right\} \leq \min \{\operatorname{rank}(A), \operatorname{rank}(B)\}$. Suppose, without loss of generality, that $c_{n-i(B)+1}=\cdots=c_{n}=0$. According to the previous lemma, for every $j \in\{1, \ldots, n-i(B)\}$, there exists $D_{j} \in F^{2 \times 2}$ similar to $D$ such that $C D_{j}$ has eigenvalues 
$c_{j}, 0$. Then, $B^{\prime}$ is similar to $B^{\prime \prime}=0_{2 i(B)-n} \oplus D_{1} \oplus \cdots \oplus D_{n-i(B)}$ and $A^{\prime} B^{\prime \prime}$ has eigenvalues $c_{1}, \ldots, c_{n}$.

Proof of Theorem 1 The necessity follows from Lemmas 2 and 3. The sufficiency follows from Lemmas 8-10.

\section{Disclosure statement}

No potential conflict of interest was reported by the authors.

\section{Funding}

The research for this paper was done within the activities of Centro de Estruturas Lineares e Combinatórias da Universidade de Lisboa (CELC) and was partially supported by the Fundação para a Ciência e Tecnologia (Portugal).

\section{References}

[1] Furtado S, Iglésias L, Silva FC. Eigenvalues of products of matrices. Linear Multilinear Algebra. 2006;54:343-353.

[2] Silva FC. The eigenvalues of the product of matrices with prescribed similarity classes. Linear Multilinear Algebra. 1993;34:269-277.

[3] Oliveira GN, Marques de Sá E, Dias da Silva JA. On the eigenvalues of the matrix $A+X B X^{-1}$. Linear Multilinear Algebra. 1977;5:119-128.

[4] Silva FC. The rank of the difference of matrices with prescribed similarity classes. Linear Multilinear Algebra. 1988;24:51-58.

[5] Furtado S, Iglésias L, Silva FC. Products of real matrices with prescribed characteristic polynomials. SIAM J. Matrix Anal. Appl. 2002;23:656-672. 